\title{
Ectopic expression of neural autoantigen in mouse liver suppresses experimental autoimmune neuroinflammation by inducing antigen-specific Tregs
}

\author{
Stefan Lüth, ${ }^{1}$ Samuel Huber, ${ }^{1}$ Christoph Schramm, ${ }^{1}$ Thorsten Buch, ${ }^{2}$ Stefan Zander, ${ }^{1}$ \\ Christine Stadelmann, ${ }^{3}$ Wolfgang Brück, ${ }^{3}$ David C. Wraith, ${ }^{4}$ \\ Johannes Herkel, ${ }^{1}$ and Ansgar W. Lohse1
}

1Department of Medicine I, University Medical Center Hamburg-Eppendorf, Hamburg, Germany. ${ }^{2}$ Department of Pathology, University Hospital Zurich, Zurich, Switzerland. ${ }^{3}$ Department of Neuropathology, Georg-August-University, Göttingen, Germany. ${ }^{4}$ Department of Pathology and Microbiology, University of Bristol, Bristol, United Kingdom.

\begin{abstract}
Tregs are important mediators of immune tolerance to self antigens, and it has been suggested that Treg inactivation may cause autoimmune disease. Therefore, immunotherapy approaches that aim to restore or expand autoantigen-specific Treg activity might be beneficial for the treatment of autoimmune disease. Here we report that Treg-mediated suppression of autoimmune disease can be achieved in vivo by taking advantage of the ability of the liver to promote immune tolerance. Expression of the neural autoantigen myelin basic protein (MBP) in the liver was accomplished stably in liver-specific MBP transgenic mice and transiently using gene transfer to liver cells in vivo. Such ectopic MBP expression induced protection from autoimmune neuroinflammation in a mouse model of multiple sclerosis. Protection from autoimmunity was mediated by MBP-specific $\mathrm{CD}^{+} \mathrm{CD}^{2} 5^{+} \mathrm{Foxp}^{+}{ }^{+}$Tregs, as demonstrated by the ability of these cells to prevent disease when adoptively transferred into nontransgenic mice and to suppress conventional $\mathrm{CD}^{+} \mathrm{CD}^{+} 5^{-} \mathrm{T}$ cell proliferation after antigen-specific stimulation with $\mathrm{MBP}$ in vitro. The generation of $\mathrm{MBP}$-specific $\mathrm{CD} 4^{+} \mathrm{CD25} 5^{+} \mathrm{Foxp}^{+}$ Tregs in vivo depended on expression of MBP in the liver, but not in skin, and occurred by TGF- $\beta$-dependent peripheral conversion from conventional non-Tregs. Our findings indicate that autoantigen expression in the liver may generate autoantigen-specific Tregs. Thus, targeting of autoantigens to hepatocytes may be a novel approach to prevention or treatment of autoimmune diseases.
\end{abstract}

\section{Introduction}

Immune tolerance to self antigens is maintained by multiple mechanisms that control potentially pathogenic autoreactive lymphocytes, including deletion, clonal anergy, or suppression by Tregs (1-3). Autoimmune disease may thus result from insufficient control of autoreactive lymphocytes $(4,5)$, and a major goal of immunotherapy for autoimmune diseases is the induction of tolerance to autoantigens by restoring regulation (6). A particularly promising way to restore self tolerance seems to be the manipulation of autoantigen-specific $\mathrm{CD} 4^{+} \mathrm{CD} 25^{+} \mathrm{Foxp} 3^{+}$ Tregs; adoptive transfer of these cells can prevent autoimmune or inflammatory conditions (6-10). In vivo, the majority of Tregs seems to be generated in the thymus (8); however, these cells may also be generated in the periphery $(9,10)$. Indeed, increasing evidence suggests that peripheral Tregs can be generated not only by peripheral expansion of thymic Tregs $(11,12)$, but also by de novo conversion from conventional CD $4^{+} \mathrm{Foxp}^{-} \mathrm{T}$ cells $(13-16)$. So far, however, it is not clear how the therapeutic potential of specific Treg generation in the periphery can be translated into clinically applicable therapies.

Nonstandard abbreviations used: CRP, C-reactive protein; K5, keratin 5; MBP, myelin basic protein.

Conflict of interest: The authors have declared that no conflict of interest exists. Citation for this article: J. Clin. Invest. 118:3403-3410 (2008). doi:10.1172/JCI32132.
The microenvironment of the liver favors immune tolerance, presumably by a combination of tolerogenic antigen-presenting cells and cytokines (17-20). We therefore hypothesized that one approach to the induction of peripheral control of autoreactive lymphocytes is the ectopic expression of autoantigen in the liver. To test our hypothesis, we used an animal model for the human neuroinflammatory disease multiple sclerosis, EAE, which is marked by $\mathrm{CD}^{+} \mathrm{T}$ cell-mediated inflammation of the central nervous system and ascending paralysis $(21,22)$. EAE was induced in susceptible B10.PL mice by autoimmunization to myelin basic protein (MBP) $(21,22)$. In B10.PL mice, which carry the $\mathrm{H}-2^{\mathrm{u}} \mathrm{MHC}$ haplotype, the encephalitogenic epitope of MBP is the aminoterminal acetylated nonameric peptide Ac1-9. Because the native Ac1-9 peptide is a poor MHC class II binder (23), we used a modified MBP in which the lysine at position 4 was replaced by tyrosine; this substitution causes greatly increased affinity to MHC class II molecules and thus more efficient lymphocyte activation (23). Ectopic expression of MBP in the liver was achieved by constitutive expression in transgenic mice that express the MBP transgene under control of the hepatocyte-specific human C-reactive protein (CRP) promoter (CRP-MBP mice; ref. 24). We also generated transgenic mice expressing MBP under control of the skin-specific keratin 5 (K5) promoter (K5-MBP mice) as controls (25). Alternatively, ectopic MBP expression in the liver was achieved by transient gene expression in hepatocytes in vivo, induced either by hydrodynamics- 
A

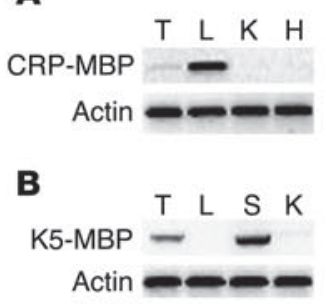

D

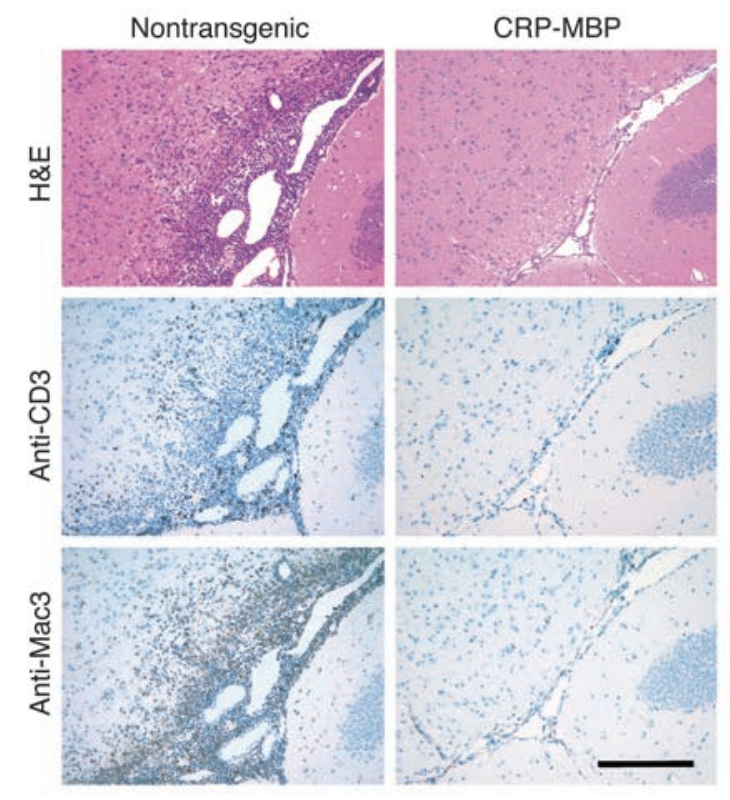

C

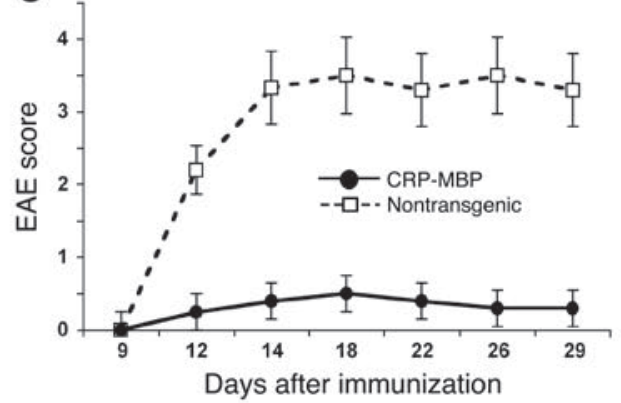

Days after immunization

CRP-MBP

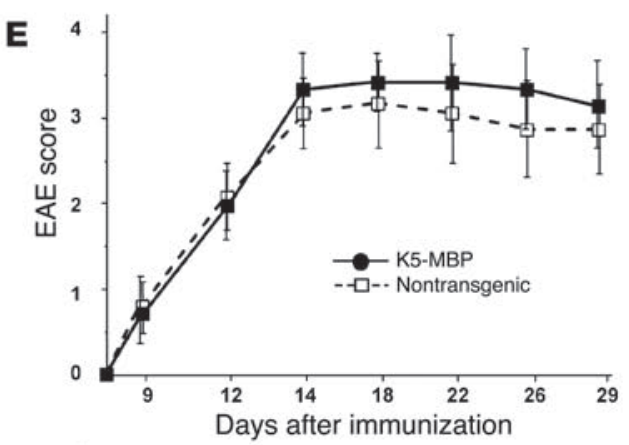

F
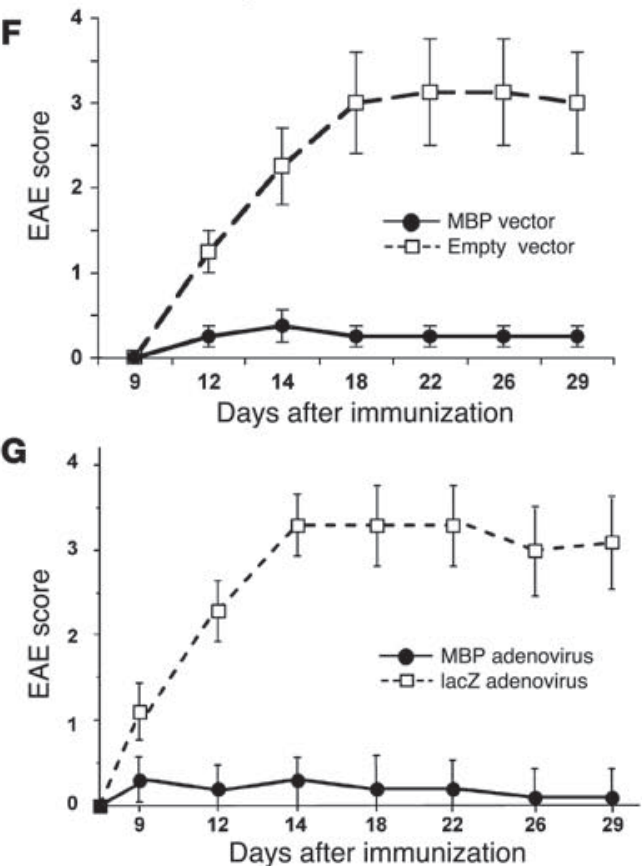

Figure 1

Ectopic expression of MBP in liver induces suppression of EAE. (A) CRP-MBP mice expressed transgenic MBP specifically in the liver (L) and weakly in the thymus $(\mathrm{T})$, but not in other organs, such as kidney $(\mathrm{K})$ and heart $(\mathrm{H})$. (B) K5-MBP mice expressed transgenic MBP specifically in the skin (S) and in the thymus, but not in liver or kidney. (C) CRP-MBP mice $(n=24)$ and nontransgenic littermates $(n=25)$ were immunized with Ac1-9 to induce EAE. Data are mean \pm SEM from 3 independent experiments. (D) Brain histology at day 18 of EAE showed dense perivascular and subpial inflammatory infiltrates in nontransgenic mice, but not in CRP-MBP mice. Staining with H\&E or immunostaining for macrophages (anti-Mac3) or T cells (anti-CD3) is shown. Scale bar: $200 \mu \mathrm{m}$. (E) K5-MBP mice $(n=11)$ and nontransgenic littermates $(n=10)$ were immunized with Ac1-9 to induce EAE. Data are mean \pm SEM from 2 independent experiments. (F) Transient hepatic MBP expression facilitated by hydrodynamics-based gene transfer with MBP-encoding vector $(n=10)$, but not by empty control vector $(n=8)$, suppressed EAE. Data are mean \pm SEM. (G) Transient hepatic MBP expression facilitated by adenoviral gene transfer of MBP $(n=10)$, but not by control adenovirus (lacZ; $n=10)$, suppressed EAE. Data are mean \pm SEM.

based gene transfer, which targets about $40 \%$ of all hepatocytes (26), or by adenoviral gene transfer (27).

We found that hepatic tolerance induced generation of MBPspecific Tregs and protection from EAE. Treg generation was thymus independent, required ectopic expression of MBP in the liver, and occurred by conversion from conventional $\mathrm{CD} 4^{+} \mathrm{CD} 25^{-} \mathrm{T}$ cells. Our findings indicate that the targeted expression of autoantigen in hepatocytes may be a novel therapeutic approach to induce autoantigen-specific $\mathrm{CD} 4^{+} \mathrm{CD} 25^{+} \mathrm{Foxp} 3^{+}$Tregs in vivo for the prevention and treatment of autoimmune diseases.

\section{Results}

To achieve ectopic expression of MBP in the liver, we generated CRP-MBP transgenic mice of FVB/N strain background express- ing the $18.5-\mathrm{kDa}$ isoform of MBP under control of the hepatocyte-specific CRP promoter; as a control, we generated K5-MBP transgenic mice that show ectopic MBP expression in the skin. Indeed, the CRP-MBP mice manifested strong expression of the transgene in the liver and weak transgene expression in the thymus (Figure 1A); the K5-MBP mice manifested strong transgene expression in both skin and thymus (Figure 1B). To establish susceptibility to EAE, the CRP-MBP and K5-MBP mice were bred with susceptible B10.PL mice, and EAE was induced in the respective F1 generations. After immunization with Ac1-9, the CRP-MBP F1 mice were protected from EAE (maximal EAE score, $0.5 \pm 0.2$; Figure 1C); the nontransgenic littermates, in contrast, developed severe EAE (maximal score, $3.5 \pm 0.6 ; P<0.05$ ). Brain histology at the time of peak EAE symptoms (i.e., 18 days after MBP immuni- 
A

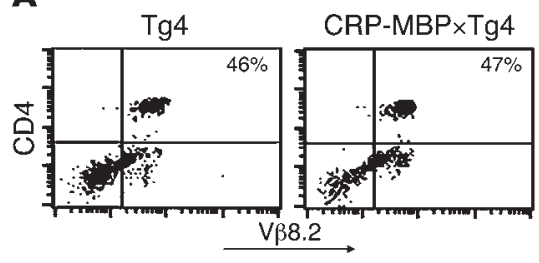

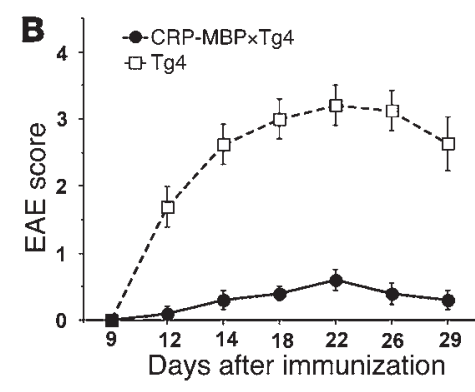

\section{Figure 2}

Protection from EAE by ectopic autoantigen expression in the liver does not depend on deletion of autoreactive lymphocytes. (A) Expression of encephalitogenic $\mathrm{T}$ cell receptor by splenic $\mathrm{CD} 4^{+} \mathrm{T}$ cells of $\mathrm{FVB} / \mathrm{N} \times \mathrm{Tg} 4$ or CRP-MBPxTg4 mice, detected by cytometry with $V \beta 8.2$-specific antibody. Percentages indicate the relative amount of peripheral $C D 4^{+} \mathrm{V} \beta 8.2^{+} \mathrm{T}$ cells. (B) CRPMBP $\times$ Tg4 mice $(n=8)$ and Tg4 littermates $(n=10)$ were immunized with Ac1-9 to induce EAE. Data are mean \pm SEM. zation) revealed that the nontransgenic mice manifested marked infiltrates of inflammatory cells, most notably macrophages and T cells, whereas CRP-MBP mice were free of cellular infiltrates (Figure $1 \mathrm{D})$. In contrast to that of CRP-MBP mice, the severity of EAE induced in K5-MBP mice (maximal score, $3.4 \pm 0.5$ ) was similar to that in nontransgenic littermates (maximal score, $3.1 \pm 0.5$; Figure $1 \mathrm{E})$. Thus, ectopic expression of MBP in the liver, but not in the skin, was associated with resistance to EAE induction.

We then asked whether transient expression of the MBP autoantigen in the liver could likewise induce protection from EAE in susceptible wild-type mice. We thus performed in vivo hydrodynamics-based MBP gene delivery to hepatocytes of adult nontransgenic mice. At 2 weeks after transfection with MBP-encoding plasmid or empty control plasmid, mice were immunized with Ac1-9 to induce EAE. Mice treated with control plasmid developed severe EAE (maximal score, $3.0 \pm 0.8$ ); in contrast, mice treated with MBP-encoding plasmid were protected from EAE (maximal score, $0.5 \pm 0.2 ; P<0.05$; Figure $1 \mathrm{~F}$ ). To confirm this observation, we also performed adenoviral gene transfer with an MBP-encoding vector or a control adenoviral vector containing the lac $Z$ gene and, 2 weeks after gene transfer, immunized with Ac1-9 to induce EAE. Adenoviral MBP gene transfer induced resistance to EAE induction (maximal score, $0.3 \pm 0.3$ ), whereas mice that received the control vector developed severe EAE (maximal score, $3.2 \pm 0.4$; $P<0.05$; Figure $1 \mathrm{G}$ ). Thus, protection from EAE was even induced by transient expression of MBP autoantigen in the liver.

To determine whether the suppression of EAE associated with ectopic MBP expression in the liver was mediated by clonal deletion of encephalitogenic T cells, we crossed CRP-MBP mice to homozygous $\mathrm{T}$ cell receptor transgenic $\mathrm{Tg} 4$ mice, which express an encephalitogenic $\mathrm{H}-2^{\mathrm{u}}$-restricted $\mathrm{V} \beta 8.2^{+} \mathrm{T}$ cell receptor that recognizes Ac1-9 (28). The resulting CRP-MBP $\times$ Tg4 doubletransgenic mice manifested peripheral $\mathrm{CD} 4^{+} \mathrm{V} \beta 8.2^{+} \mathrm{T}$ cells at numbers similar to those of $\mathrm{Tg} 4$ single-transgenic littermates (Figure 2A). Thus, the encephalitogenic T cells had not been deleted in CRP-MBP mice. Nevertheless, after immunization to Ac1-9, the CRP-MBP $\times$ Tg 4 mice were protected from EAE (maximal score, $0.6 \pm 0.16$ ), in contrast to $\mathrm{FVB} / \mathrm{N} \times \mathrm{Tg} 4$ mice (maximal score, $3.2 \pm 0.3 ; P<0.0001$; Figure $2 \mathrm{~B}$ ). This protection from EAE was also not caused by downregulation of autoreactive $\mathrm{T}$ cell receptors (29): $\mathrm{T}$ cells from CRP-MBP $\times \mathrm{Tg} 4$ mice had surface expression of CD4 and V $\beta 8.2$ similar to that of T cells from FVB/ $\mathrm{N} \times \mathrm{Tg} 4$ mice (Figure 2A). Thus, the MBP-specific T cells of CRPMBP mice were not different from those of nontransgenic mice in frequency or $\mathrm{T}$ cell receptor expression.

Next, we investigated whether protection from EAE in CRP-MBP mice is mediated by active suppression. We adoptively transferred $10^{6}$ spleen cells from CRP-MBP mice into nontransgenic mice; as a control, we transferred $10^{6}$ splenocytes from nontransgenic mice. Mice that received splenocytes from CRP-MBP donors were protected from EAE (maximal score, $0.5 \pm 0.2$ ), whereas mice that received splenocytes from nontransgenic donors were not protected and developed EAE symptoms (maximal score, $3.0 \pm 0.4$; $P<0.05$; data not shown). To further characterize the cell type that transferred resistance to EAE, we sorted splenocytes from CRP-MBP mice according to their staining for CD4 and CD8 and transferred $10^{5}$ cells of the $\mathrm{CD}^{+}, \mathrm{CD}^{+}$, or $\mathrm{CD}^{-} \mathrm{CD} 8^{-}$fractions into nontransgenic recipient mice. $\mathrm{CD}^{+}$cells from CRP-MBP mice (maximal score, $1.0 \pm 0.4$ ), but not from nontransgenic mice (maximal score, $3.3 \pm 0.6$ ), transferred resistance to EAE effectively; transferred $\mathrm{CD}^{+}{ }^{+}$or $\mathrm{CD}^{-}{ }^{-} \mathrm{CD} 8{ }^{-}$cells from CRP-MBP mice did not protect from EAE (maximal score, $>3.0 ; P<0.005$; Figure 3A).

In order to determine whether protection from EAE by transferred $\mathrm{CD}^{+} \mathrm{T}$ cells depends on $\mathrm{CD} 4^{+} \mathrm{CD} 25^{+}$Tregs, we sorted splenic CD $4^{+} \mathrm{T}$ cells from CRP-MBP mice into a CD $25^{+}$fraction and a CD25- fraction and transferred $10^{5}$ cells of each fraction into wild-type recipient mice, which were subsequently immunized to MBP. While transfer of $\mathrm{CD} 4^{+} \mathrm{CD} 25^{+} \mathrm{T}$ cells protected from EAE (maximal score, $0.5 \pm 0.2$ ), transfer of $\mathrm{CD} 4^{+} \mathrm{CD} 25^{-} \mathrm{T}$ cells did not (maximal score, $3.3 \pm 0.4 ; P<0.05$; Figure $3 \mathrm{~B}$ ). Thus, protection from EAE in CRP-MBP mice was conferred by a CD25+ fraction of splenic CD4 $\mathrm{T}$ cells. We then confirmed that the protective $\mathrm{CD} 4^{+} \mathrm{CD} 25^{+} \mathrm{T}$ cells were indeed Tregs by demonstrating their expression of Foxp3 (Figure 3C).

Because tolerance to peripheral autoantigens can be induced by their thymic expression (30), and the CRP-MBP mice manifested weak thymic expression of the transgene (Figure 1A), we therefore investigated whether the protective $\mathrm{CD} 4^{+} \mathrm{CD} 25^{+} \mathrm{Foxp} 3^{+}$Tregs from CRP-MBP mice are generated in the thymus or in the periphery. First, we bypassed thymic tolerance induction by neonatal thymectomy of CRP-MBP mice, followed by engraftment of embryonic thymus from nontransgenic mice and - after 6.5-Gy irradiation - reconstitution with bone marrow from nontransgenic mice. As a control, the same procedure was performed in nontransgenic mice. The thymectomized CRP-MBP mice were protected from EAE (maximal score, $0.3 \pm 0.2$ ), but the thymectomized nontransgenic mice developed severe EAE (maximal score, $3.3 \pm 0.5 ; P<0.05$; Figure $3 \mathrm{D}$ ). Thus, protection from EAE did not seem to depend on expression of MBP in the thymus. To confirm this interpretation, we isolated thymic $\mathrm{CD} 8{ }^{-} \mathrm{CD} 4^{+} \mathrm{CD} 25^{+}$Tregs from the thymus of CRP-MBP or nontransgenic mice, transferred $10^{5}$ thymic Tregs into nontransgenic recipients, and assessed the cells' potential to suppress EAE. Thymic Tregs from both CRP-MBP and nontransgenic mice were inefficient in suppression of EAE (maximal score, $>3.0$; Figure $3 \mathrm{E}$ ). Since $10^{5}$ peripheral Tregs (Figure 3B), but not $10^{5}$ thymic Tregs from CRP-MBP mice (Figure $3 \mathrm{E}$ ), efficiently pro- 

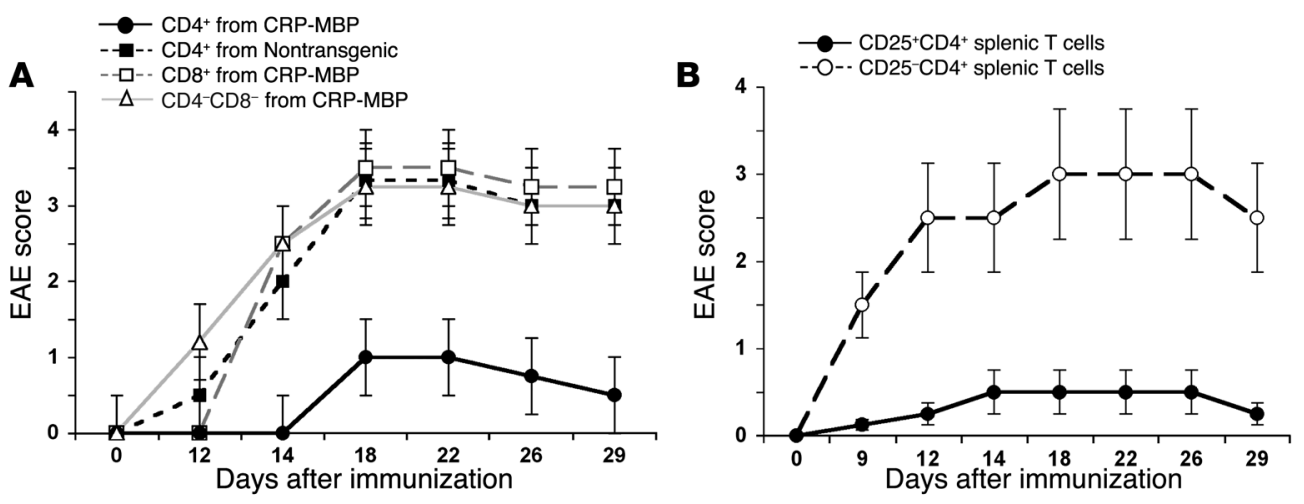

$2 \times 10^{6} \mathrm{CFSE}^{+} \mathrm{CD}^{+} \mathrm{CD}^{2} 5^{-} \mathrm{T}$

cells into nontransgenic or CRP-MBP mice (Figure 4A). At 7 days after transfer, we reisolated the $\mathrm{CFSE}^{+} \mathrm{T}$ cells from spleens and livers of the recipient mice (Figure $4 \mathrm{~B}$ ) and further transferred the recovered $\mathrm{CFSE}^{+} \mathrm{Tg} 4 \mathrm{~T}$ cells into nontransgenic recipients $\left(2 \times 10^{4}\right.$ cells/mouse $)$, which were then immunized with Ac1-9 to induce EAE (Figure $4 A)$. We transferred only those $\mathrm{CFSE}^{+}$cells that could be certainly distinguished from the endogenous $\mathrm{CFSE}^{-}$cells by their fluorescence intensity, i.e., cells that underwent no more than 5 divisions (Figure 4B). The recipients of $\mathrm{Tg} 4 \mathrm{~T}$ cells that were placed in nontransgenic mice developed severe EAE (maximal score, $3.4 \pm 0.2$ ), whereas the $\mathrm{Tg} 4 \mathrm{~T}$ cells placed in CRP-MBP mice conferred protection from
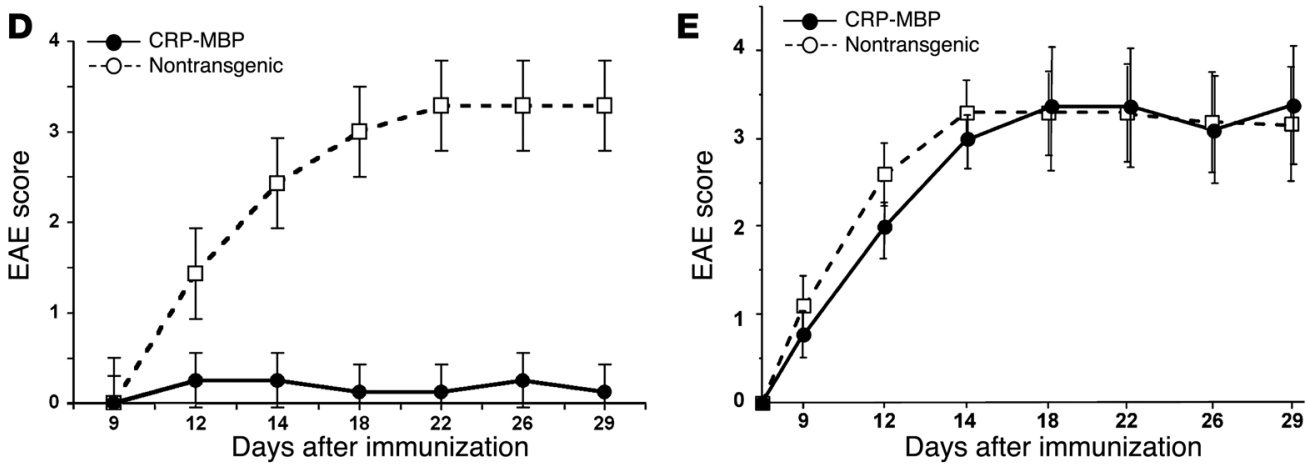

Figure 3

Liver-induced protection from EAE is mediated by peripheral Tregs. (A) Suppression of EAE by adoptive transfer into wild-type mice of $10^{5}$ splenic $\mathrm{CD}^{+}$cells - but not $\mathrm{CD}^{+}$or $\mathrm{CD}^{-}{ }^{-C D} 8^{-}$cells - from CRP-MBP mice, or of $\mathrm{CD}^{+}{ }^{+} \mathrm{T}$ cells from nontransgenic mice. Data are mean $\pm \mathrm{SEM}$. (B) Protection of wild-type mice from EAE by adoptive transfer of $10^{5}$ splenic CD25+CD4+ $T$ cells from CRP-MBP mice, but not by transfer of $10^{5}$ splenic CD25-CD4+ T cells from CRP-MBP mice. Data are mean \pm SEM. (C) Spleen cells from CRPMBP mice were gated for expression of $C D 4$ and CD25; percentage indicates $C D 4{ }^{+} \mathrm{CD} 25^{+}$proportion of the total splenocytes. Expression of Foxp3 in $\mathrm{CD} 4{ }^{+} \mathrm{CD} 25^{+}$and $\mathrm{CD} 4{ }^{+} \mathrm{CD} 25^{-} \mathrm{T}$ cells is shown at right; percentages indicate Foxp3 ${ }^{+}$proportion of the splenic $\mathrm{CD} 4{ }^{+} \mathrm{CD} 25^{+}$and $\mathrm{CD} 4{ }^{+} \mathrm{CD} 25^{-}$cells. (D) Neonatal thymectomy and 6.5-Gy irradiation of CRP-MBP mice $(n=14)$ or nontransgenic littermates $(n=12)$, followed by reconstitution with bone marrow and embryonic thymus from wild-type mice, was performed to bypass possible thymic immune tolerance to MBP before EAE induction. Data are mean \pm SEM. (E) Transfer of $10^{5} \mathrm{CD} 8^{-} \mathrm{CD} 4^{+} \mathrm{CD} 25^{+}$ thymic Tregs from CRP-MBP or nontransgenic mice ( $n=10$ per group) into wild-type mice failed to protect the recipient mice from subsequent induction of EAE. Data are mean $\pm \mathrm{SEM}$.

tected from EAE, it is highly unlikely that the protective Tregs were induced in the thymus.

To further confirm that the protective Tregs in CRP-MBP mice were generated in the periphery rather than in the thymus, we isolated naive $\mathrm{CD} 4^{+} \mathrm{CD} 25^{-} \mathrm{CD} 62 \mathrm{~L}^{\text {high }} \mathrm{T}$ cells from the spleens of $\mathrm{Tg} 4$ mice (>99\% Foxp3 $3^{-}$), labeled these cells with CFSE, and transferred
EAE (maximal score, $1.4 \pm 0.3$; $P<0.05$; Figure 4C). Thus, the transferred naive $\mathrm{CD}^{+}$Ac1-9specific $T$ cells appeared to acquire the ability to suppress EAE by passage through CRP-MBP mice. Peripheral generation of Tregs is known to occur by conversion from conventional $\mathrm{CD} 4^{+} \mathrm{CD} 25^{-} \mathrm{T}$ cells (9); thus, to confirm that the protective $\mathrm{CFSE}^{+} \mathrm{T}$ cells obtained by passage through CRP-MBP mice were generated by conversion to Tregs in vivo, we analyzed their Foxp 3 expression. Indeed, the $\mathrm{CFSE}^{+}$cells recovered from CRP-MBP mice, but not from nontransgenic mice, exhibited increased Foxp3 expression (8.1\% and $1 \%$, respectively; Figure 4D). Foxp3 staining of the $\mathrm{CD}^{+} \mathrm{CFSE}^{-} \mathrm{T}$ cells served as internal staining control. Because, unlike thymic Treg generation, the peripheral conversion of Tregs from conventional $\mathrm{T}$ cells critically depends on TGF- $\beta$ (9), we sought to confirm that the conversion of naive $\mathrm{CFSE}^{+} \mathrm{T}$ cells to protective CFSE ${ }^{+} \mathrm{Foxp}^{+} \mathrm{T}$ cells in CRP-MBP mice was TGF- $\beta$ dependent. Therefore, we isolated $\mathrm{CD} 4^{+} \mathrm{CD} 25^{-} \mathrm{V} \beta 8.2^{+}$ $\mathrm{T}$ cells from the spleens of $\mathrm{Tg} 4 \times \mathrm{hCD} 2-\Delta \mathrm{kT} \beta \mathrm{RII} F 1$ mice, which manifest impaired TGF- $\beta$ signaling (31). After labeling with CFSE 
A

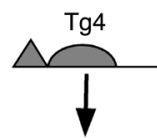

CD4+CD25-CD62L high naive MBP-specific $T$ cells
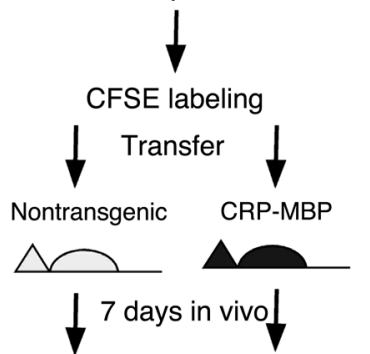

Recovery of $\mathrm{CFSE}^{+}$cells

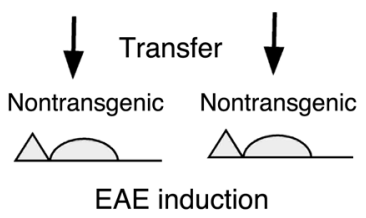

B

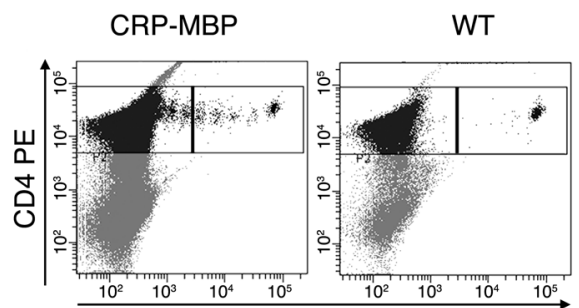

CFSE

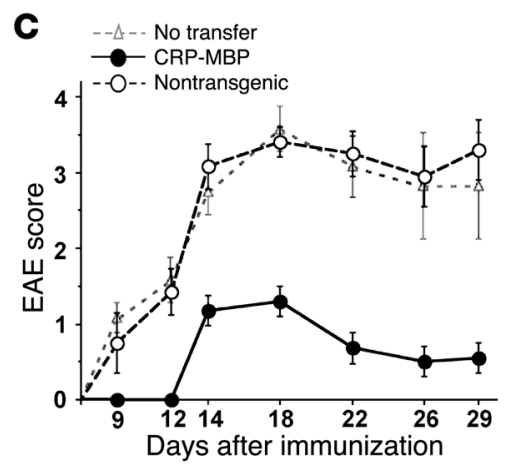

D
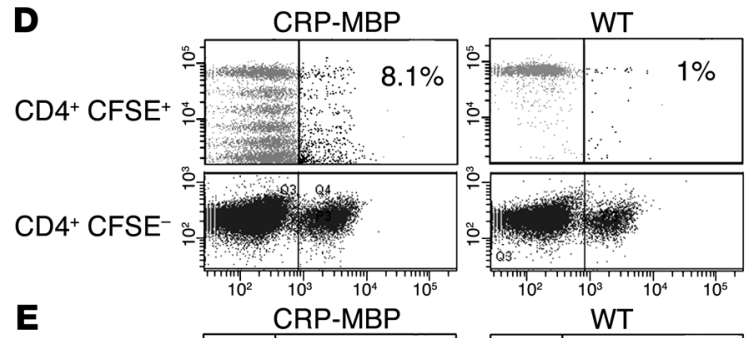

E

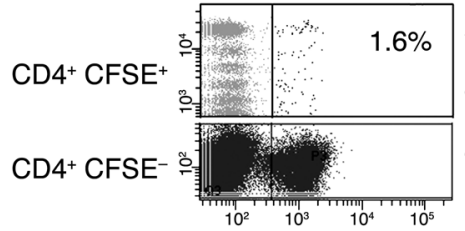

F K5-MBP

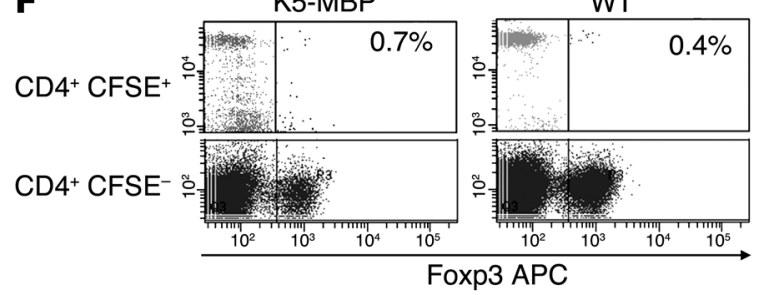

Figure 4

Protective Tregs are generated by TGF- $\beta$-dependent peripheral conversion from naive CD4+CD25- T cells. (A) Experimental procedure for assessing in vivo conversion of naive MBP-specific T cells to Tregs. Naive splenic T cells from Tg4 mice were labeled with CFSE and transferred to nontransgenic or CRP-MBP mice; after 7 days, they were recovered again for transfer to nontransgenic mice, in which EAE was induced. (B) Analysis of transferred CFSE ${ }^{+}$and residual $\mathrm{CFSE}^{-}$cells retrieved from CRP-MBP or nontransgenic mice. The CD4 ${ }^{+} \mathrm{CFSE}{ }^{+}$cells in the respective upper right gates were selected for transfer into wild-type mice. (C) CD4+CFSE+ cells retrieved from nontransgenic $(n=10)$ or $\mathrm{CRP}-\mathrm{MBP}$ $(n=11)$ mice were transferred to nontransgenic recipient mice $\left(2 \times 10^{4}\right.$ cells per mouse), in which EAE was induced. Mice without cell transfer $(n=8)$ served as a control. Data are mean \pm SEM. (D-F) Analysis of CFSE-labeled T cells ( $\mathbf{D}$ and F) and CFSE-labeled TGF- $\beta$-insensitive T cells $(\mathbf{E})$. Cells were retrieved from spleen and liver 7 days after transfer to CRP-MBP (D and E), K5-MBP (F), or nontransgenic mice. Percent Foxp ${ }^{+}{ }^{+} \mathrm{FSE}^{+} \mathrm{T}$ cells is indicated.

dye, the TGF- $\beta$-insensitive T cells were transferred to nontransgenic mice or CRP-MBP mice, recovered from spleens and livers after 7 days, and analyzed for Foxp3 expression (Figure 4E). In contrast to the TGF- $\beta$-responsive T cells (Figure 4D), the TGF- $\beta$ insensitive $T$ cells did not acquire marked Foxp3 expression when transferred to nontransgenic or CRP-MBP mice (1.3\% and $1.6 \%$, respectively; Figure 4E). These findings indicate that $\mathrm{MBP}$ expression in the livers of CRP-MBP mice induces the conversion of conventional MBP-specific T cells to Foxp $3^{+}$Tregs and that this conversion is dependent on TGF- $\beta$. However, the transferred CFSE ${ }^{+}$ $T$ cells apparently were not efficiently stimulated to proliferate in nontransgenic mice, as indicated by the absence of CFSE dilution in contrast to the evident proliferation of $\mathrm{CFSE}^{+} \mathrm{T}$ cells that were transferred to CRP-MBP mice (Figure 4, D and E). It was possible that a lack of stimulation could explain the absence of conversion to Foxp3 $3^{+}$Tregs in nontransgenic mice. Therefore, we studied whether transfer of naive $\mathrm{CFSE}^{+} \mathrm{Tg} 4 \mathrm{~T}$ cells to K5-MBP mice could induce proliferation and Foxp3 expression (Figure 4F). The $\mathrm{CFSE}^{+} \mathrm{T}$ cells transferred to K5-MBP mice were stimulated in vivo, as indicated by the dilution of CFSE ( $51 \%$ nonproliferated cells, compared with 53\% after transfer to CRP-MBP mice; Figure 4D; and $88 \%$ after transfer to nontransgenic mice; Figure $4 \mathrm{~F}$ ). However, $\mathrm{CFSE}^{+} \mathrm{T}$ cells that were transferred to K5-MBP mice did not exhibit increased Foxp3 expression compared with those transferred to nontransgenic mice $(0.7 \%$ and $0.4 \%$, respectively; Figure $4 \mathrm{~F})$. Thus, the in vivo conversion of $\mathrm{CFSE}^{+} \mathrm{T}$ cells to Foxp $3^{+}$Tregs was not induced by their peripheral stimulation unless the stimulating antigen was expressed in the liver.

Although these findings indicated that ectopic expression of MBP in the liver induced the conversion of conventional T cells into Tregs, there was no apparent general increase in Treg number in CRP-MBP mice. Indeed, the frequency of $\mathrm{CD} 4^{+} \mathrm{CD} 25^{+} \mathrm{Foxp} 3^{+} \mathrm{T}$ cells (about 9\%) did not significantly differ between CRP-MBP and nontransgenic mice. Thus, it was possible that the frequency of MBP-specific Tregs or their suppressive activity was selectively increased in CRP-MBP mice. To confirm that MBP expression in the liver induces selective expansion of MBP-specific Tregs, we compared the capacity of $\mathrm{CD} 4^{+} \mathrm{CD} 25^{+} \mathrm{T}$ cells from CRP-MBP mice to suppress the effector responses of $\mathrm{T}$ cells from $\mathrm{Tg} 4$ mice with that of nontransgenic littermates (Figure 5). Tregs from CRP-MBP mice were as effective as Tregs from nontransgenic mice in suppressing conventional $\mathrm{CD} 4^{+} \mathrm{CD} 25^{-}$ effector $\mathrm{T}$ cells in response to nonspecific stimulation with antibody to CD3 (Figure 5A). In contrast, as few as $5 \times 10^{3}$ Tregs from CRPMBP mice completely suppressed the proliferation of $10^{5}$ effector T cells after Ac1-9 stimulation, whereas at least $5 \times 10^{4}$ Tregs from nontransgenic mice were required to obtain a similar degree of suppression (Figure 5B). These findings indicate that MBP expression in the liver induced a selective increase in MBP-specific Treg activity.

\section{Discussion}

Immunotherapy for autoimmune disease aims at restoring a state of tolerance to the self antigens that drive autoimmune disease. 
A
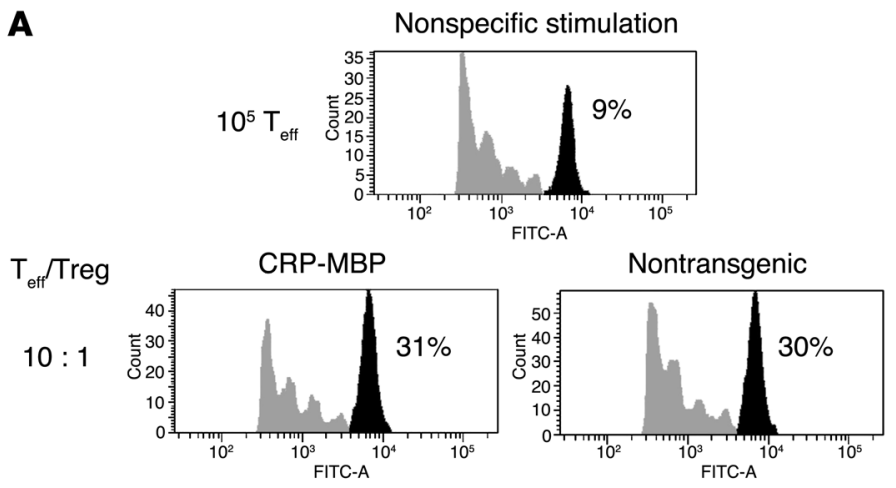

$2: 1$

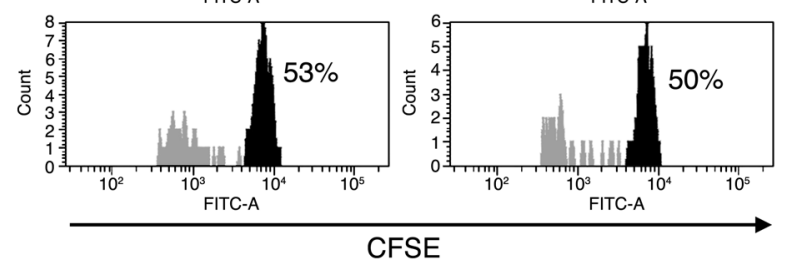

B
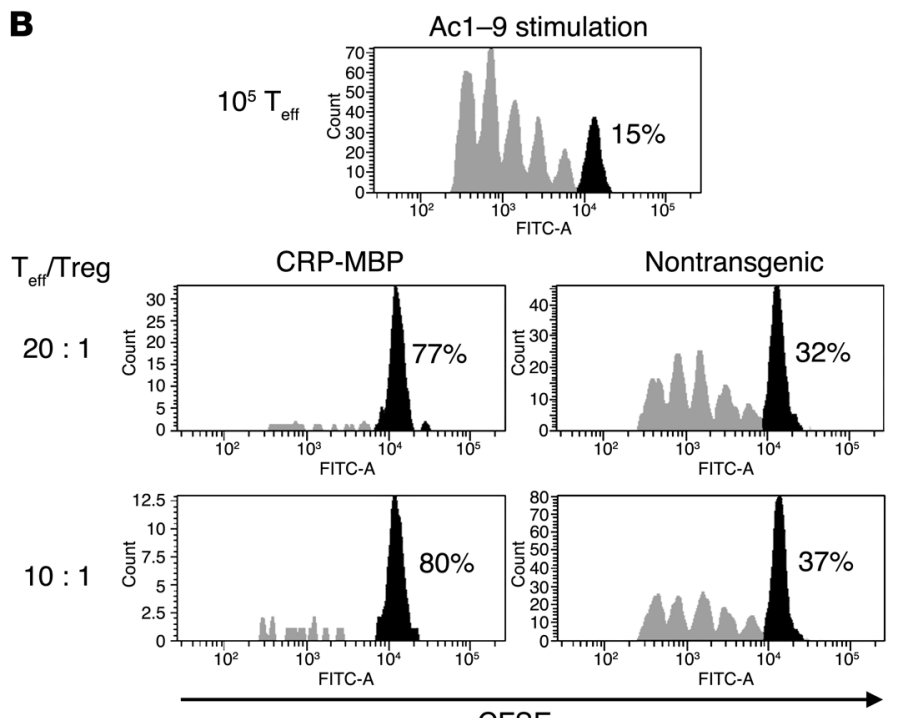

CFSE

Our findings indicate that the targeted expression of autoantigens in liver cells may be a novel approach to induce active tolerance to autoantigens, which may serve as therapy for autoimmune disease. Indeed, ectopic expression of MBP in liver, but not in skin, suppressed subsequent EAE induction, even when expressed transiently (Figure 1). This protection was not mediated by clonal deletion (Figure 2), but by peripheral Tregs (Figure 3), which seemed to be generated by de novo TGF- $\beta$-dependent conversion from conventional $\mathrm{CD}^{+} \mathrm{CD} 25^{-} \mathrm{T}$ cells (Figure 4), resulting in selective expansion of MBP-specific Treg activity (Figure 5).

How exactly these Tregs were induced by autoantigen expression in the liver is currently not clear. However, it is unlikely that central tolerance was involved in protection from EAE, because (a) transient MBP expression in liver induced protection; (b) there was no apparent deletion or receptor downregulation (Figure 2); (c) neonatal thymectomy did not abrogate protection (Figure 3D); (d) thymic Tregs did not confer protection (Figure 3E); (e) K5-MBP mice were not protected (Figure 4F); and (f) peripheral TGF- $\beta$-dependent conversion from conventional $\mathrm{T}$ cells induced protective Tregs (Fig-

\section{Figure 5}

Selective expansion of autoantigen-specific Tregs in mice expressing the autoantigen ectopically in the liver. Conventional CD25$\mathrm{CD}^{+}{ }^{+}$effector $\mathrm{T}$ cells $\left(\mathrm{T}_{\text {eff }}\right)$ from $\mathrm{Tg} 4$ mice were labeled with CFSE and activated in the absence or presence of CD25+CD4+ Tregs from CRP-MBP or nontransgenic mice at the indicated ratios. (A) Tregs of CRP-MBP and nontransgenic mice showed an equal capacity to suppress after nonspecific stimulation with antibody to CD3. (B) Tregs from CRP-MBP mice showed greatly increased efficacy to suppress after being stimulated with Ac1-9 compared with Tregs from nontransgenic mice, indicating selective expansion of MBP-specific Tregs in CRP-MBP mice. Percentages indicate the proportion of cells that did not proliferate (black peaks).

ure 4). Nevertheless, we cannot formally prove thus far that the conversion to Tregs was induced by cells in the liver, although it is clear that suppression of neuroinflammation occurred only when neural autoantigen was ectopically expressed in the liver, not in the skin (Figures 1 and 4).

The liver contains various cell types that can interact with $\mathrm{CD}^{+} \mathrm{T}$ cells and have known abilities to regulate immune responses, mostly by way of immune deviation. These cell types include Kupffer cells $(32,33)$, sinusoidal endothelial cells $(19,20)$, hepatic stellate cells $(34,35)$, and hepatocytes $(36,37)$. Although it was previously shown that some hepatic cell types can regulate the suppressive activity of Tregs (38), it is thus far not known whether any of these cells are able to convert non-Tregs to Tregs. However, hepatocytes, to which expression of the autoantigen was targeted, do not express MHC class II molecules constitutively (36); thus, it is possible that not hepatocytes themselves, but instead another type of antigen-presenting cell in the liver or in the draining lymph nodes, induced the conversion to Tregs after sequestration of MBP released from the hepatocytes.

Regardless of what cell type induced the conversion to Tregs, there are indications that the mechanisms of liver-induced conversion could be distinct from those of nonhepatic conversion. First, this conversion appeared to withstand contextual inflammation in response to adenoviral gene delivery (Figure $1 \mathrm{G})$. Notably, adenoviral infection is associated with increased interleukin- 6 levels, and interleukin- 6 is known to abrogate TGF- $\beta$-dependent conversion in favor of generating Th17 cells (39). Second, at least in vivo, the liver-induced conversion into Tregs appeared to involve not initial clonal expansion, but rather immediate acquisition of the phenotypic Treg marker Foxp3, even in nonexpanded cells (Figure 4D). Therefore, the cell types and molecular signals responsible for hepatic conversion of non-Tregs to Tregs should be further investigated. Indeed, elucidating the mechanisms of Treg generation by hepatic antigens may also help to explain the causes of tolerance loss in autoimmune liver disease; the liver can be the target of various aggressive autoimmune diseases of as yet unknown etiologies. Impaired TGF- $\beta$ signaling in T cells has previously been found to increase the susceptibility to autoimmune hepatitis (31); here, we found that the liver-induced conversion of conventional T cells to Tregs was TGF- $\beta$ dependent (Figure 4E). Thus, the susceptibility to autoimmune liver disease may be related to defective hepatic generation of Tregs. Indeed, a relative Treg deficiency has been reported to occur in patients with autoimmune liver disease $(40,41)$. However, such flawed generation of Tregs may only predispose - not cause - autoimmune liver disease; it remains unclear 
what brings about the differentiation and activation of autoaggressive lymphocytes in autoimmune liver disease.

It has previously been reported that antigen-specific Tregs may also be expanded by neurons in vivo (42), or by antigen-pulsed dendritic cells in vitro (43). Although it remains to be seen whether these prior approaches or the one described here can be developed into practical therapies for human autoimmune disease, the method of Treg expansion described here may be promising because gene delivery to hepatocytes is becoming a realistic therapeutic option (27). Moreover, the MBP-specific Tregs induced by hepatic MBP expression featured extraordinary suppressive efficacy, both in abrogating the proliferation even of a 20 -fold excess of MBPstimulated conventional $T$ cells in vitro (Figure 5) and in suppressing EAE in vivo after the transfer of as few as $2 \times 10^{4}$ converted $\mathrm{T}$ cells (Figure 4). Of note, even transient hepatic expression of autoantigen induced robust control of autoreactive lymphocytes (Figure 1, F and G). Thus, targeting of autoantigen expression to the liver may indeed be an attractive approach to induce active tolerance to autoantigens and protect from autoimmune disease, and the usefulness of this approach to serve as therapy for human autoimmune disease should be explored.

\section{Methods}

Mice. All mice were bred and kept in a specific pathogen-free animal facility at Johannes Gutenberg University (Mainz, Germany) or at the University Medical Centre Hamburg-Eppendorf. The experiments were approved by the institutional review boards Bezirksregierung Rheinhessen (Neustadt, Germany) and Behörde für Soziales, Familie, Gesundheit, und Verbraucherschutz (Hamburg, Germany). Complementary DNA encoding a MBP splice variant of $18.5 \mathrm{kDa}$, in which the lysine at position 4 was replaced by tyrosine, was generated by PCR with the following primers: sense, 5'-ACCCCCGGGATGGCATCACAGTACAGACCCTCA-3'; antisense, 5'-ACCCCCGGGTCAGCGTCTCGCCATGGGAGATCC-3'. CRP-MBP mice were generated according to standard procedures (44) by microinjection of the generated complementary DNA constructs ligated to the human CRP promoter (24) into fertilized FVB oocytes. Of the 5 founder lines obtained, 4 expressed MBP in the liver and showed protection from EAE. As control, $\mathrm{K} 5-\mathrm{MBP}$ mice were generated by ligating the MBP construct to the K5 promoter (25); 2 founder lines were obtained that expressed MBP in the skin. Alternatively, ectopic expression of MBP in mouse liver was achieved by hydrodynamics-based gene transfer of MBP gene, cloned into pcDNA3.1 plasmid (Invitrogen) as described previously (26), or by adenoviral gene transfer of MBP gene cloned into a type 5 adenoviral vector, provided by J. Prieto (University of Navarra, Pamplona, Spain).

$E A E$ induction. Age- and sex-matched mice were inoculated subcutaneously at the base of the tail with $200 \mu \mathrm{g}$ Ac1-9 (Sigma-Aldrich) in $0.1 \mathrm{ml}$ of a sonicated emulsion of an equal volume of complete Freund's adjuvant and PBS containing $4 \mathrm{mg} / \mathrm{ml}$ heat-killed Mycobacterium tuberculosis, strain H37RA
(Difco). One day after immunization, $200 \mathrm{ng}$ pertussis toxin (Sigma-Aldrich) was administered intraperitoneally in $0.5 \mathrm{ml}$ PBS. Thereafter, EAE was monitored daily for up to 30 days. The severity of EAE was scored as follows: 1, flaccid tail; 2, partial hindlimb paralysis and impaired righting reflex; 3 , total hindlimb paralysis; 4 , fore- and hindlimb paralysis; 5 , moribund.

Histopathology and immunohistochemistry. For histopathological analysis of the CNS, brain and spinal cord sections were stained with Luxol Fast Blue or immunostained with the indicated rat monoclonal antibodies and biotinylated secondary antibody (GE Healthcare) followed by ExtrAvidin-Peroxidase (Sigma-Aldrich) and developed with DAB (Sigma-Aldrich) according to the manufacturers' instructions.

Adoptive transfer of $T$ cell subsets. Purified $\mathrm{CD} 4^{+}$and $\mathrm{CD} 8^{+}$or $\mathrm{CD} 4^{+} \mathrm{CD} 25^{+}$ $\mathrm{T}$ cells from the spleens of MBP transgenic mice or nontransgenic mice were separated by MACS (Miltenyi) according to the manufacturer's instructions; the purity of the separated cells was above $95 \%$ as controlled by a FACSscan or FACScanto (BD Biosciences). CD $4{ }^{+} \mathrm{CD} 25^{-} \mathrm{CD} 62 \mathrm{~L}^{\text {high }}$ non-Tregs or $\mathrm{CD}^{+} \mathrm{CD} 25^{-} \mathrm{V} \beta 8.2^{+} \mathrm{T}$ cells were obtained with the MultiSort MACS kit from Miltenyi and were greater than $99 \%$ Foxp3 $^{-}$, as controlled by fluorescence-activated cell sorting. $\mathrm{CD} 4{ }^{+} \mathrm{CD} 8-\mathrm{CD} 25^{+}$thymic Tregs were obtained by sorting in a FACSaria (BD Biosciences). Cell populations were then suspended in PBS and injected intraperitoneally on the day of EAE induction. $\mathrm{CFSE}^{+}$cells were obtained by presorting for $\mathrm{CD} 4$ expression by MACS, followed by CFSE sorting in a FACSaria (BD Biosciences).

$T$ cell suppressor assays. Suppressor assays were performed essentially as described previously (45). Proliferation was determined by CFSE labeling of effector $\mathrm{T}$ cells, detected by flow cytometry, as described previously (45). Foxp3 staining was determined by flow cytometry with APC-labeled Foxp3 antibody (eBioscience).

Statistics. A $P$ value less than 0.05 was considered significant. Statistical significance of differences between data sets was determined by the Mann-Whitney test.

\section{Acknowledgments}

This study was supported by the Deutsche Forschungsgemeinschaft (SFB 548, SCHR 781/1-2, and HE 3532/2-1) and the Wellcome Trust.

Received for publication March 14, 2007, and accepted in revised form August 20, 2008.

Address correspondence to: Johannes Herkel, Department of Medicine I, University Medical Centre Hamburg-Eppendorf, Martinistr. 52, 20246 Hamburg, Germany. Phone: 49-40-428039736; Fax: 49-40-428038014; E-mail: jherkel@uke-uni-hamburg.de.

Johannes Herkel and Ansgar W. Lohse are co-senior authors.

Stefan Lüth and Samuel Huber contributed equally to this work.
1. Hogquist, K.A., Baldwin, T.A., and Jameson, S.C. 2005. Central tolerance: learning self-control in the thymus. Nat. Rev. Immunol. 5:772-782.

2. Goodnow, C.C., Sprent, J., Fazekas de St. Groth, B., and Vinuesa, C.G. 2005. Cellular and genetic mechanisms of self tolerance and autoimmunity. Nature. 435:590-597.

3. Jonuleit, H., and Schmitt, E. 2003. The regulatory T cell family: distinct subsets and their interrelations. J. Immunol. 171:6323-6327.

4. Cohen, I.R. 2000. Tending Adam's garden: evolving the cognitive immune self. Academic Press. New York, New York, USA. 266 pp.

5. Viglietta, V., Baecher-Allan, C., Weiner, H.L., and
Hafler, D.A. 2004. Loss of functional suppression by CD4+CD25+ regulatory $\mathrm{T}$ cells in patients with multiple sclerosis. J. Exp. Med. 199:971-979.

6. von Herrath, M.G., and Harrison, L.C. 2003. Antigen-induced regulatory $\mathrm{T}$ cells in autoimmunity. Nat. Rev. Immunol. 3:223-232.

7. Fontenot, J.D., Gavin, M.A., and Rudensky, A.Y. 2003. Foxp3 programs the development and function of CD $4+\mathrm{CD} 25+$ regulatory T cells. Nat. Immunol. 4:330-336.

8. Sakaguchi, S. 2005. Naturally arising Foxp3-expressing $\mathrm{CD} 25+\mathrm{CD} 4+$ regulatory $\mathrm{T}$ cells in immunological tolerance to self and non-self. Nat. Immunol. 6:345-352.
9. Wan, Y.Y., and Flavell, R.A. 2006. The roles for cytokines in the generation and maintenance of regulatory T cells. Immunol. Rev. 212:114-130.

10. Huber, S., and Schramm, C. 2006. TGF-beta and CD4+CD25+ regulatory T cells. Front. Biosci. 11:1014-1023.

11. Gavin, M.A., Clarke, S.R., Negrou, E., Gallegos, A., and Rudensky, A. 2002. Homeostasis and anergy of CD4(+)CD25(+) suppressor T cells in vivo. Nat. Immunol. 3:33-41.

12. Walker, L.S., Chodos, A., Eggena, M., Dooms, H., and Abbas, A.K. 2003. Antigen-dependent proliferation of CD4+ CD25+ regulatory T cells in vivo. J. Exp. Med. 198:249-258. 
13. Liang, S., et al. 2005. Conversion of CD4+ CD25cells into CD4+CD25+ regulatory $\mathrm{T}$ cells in vivo requires $\mathrm{B} 7$ costimulation, but not the thymus. J. Exp. Med. 201:127-137.

14. Knoechel, B., Lohr, J., Kahn, E., Bluestone, J.A., and Abbas, A.K. 2005. Sequential development of interleukin 2-dependent effector and regulatory $\mathrm{T}$ cells in response to endogenous systemic antigen. J. Exp. Med. 202:1375-1386.

15. Kretschmer, K., et al. 2005. Inducing and expanding regulatory $\mathrm{T}$ cell populations by foreign antigen. Nat. Immunol. 6:1219-1227.

16. Quintana, F.J., et al. 2008. Control of T(reg) and $\mathrm{T}(\mathrm{H}) 17$ cell differentiation by the aryl hydrocarbon receptor. Nature. 453:65-71.

17. Crispe, I.N. 2003. Hepatic T cells and liver tolerance. Nat. Rev. Immunol. 3:51-62.

18. Limmer, A., et al. 1998. Failure to induce organspecific autoimmunity by breaking of tolerance: importance of the microenvironment. Eur. J. Immunol. 28:2395-2406.

19. Knolle, P.A., et al. 1999. Induction of cytokine production in naïve CD4 ( + T cells by antigen-presenting murine liver sinusoidal endothelial cells but failure to induce differentiation toward Th1 cells. Gastroenterology. 116:1428-1440.

20. Limmer, A., et al. 2000. Efficient presentation of exogenous antigen by liver endothelial cells to $\mathrm{CD} 8+\mathrm{T}$ cells results in antigen-specific $\mathrm{T}$ cell tolerance. Nat. Med. 6:1348-1354.

21. Zamvil, S.S., and Steinman, L. 1990. The T lymphocyte in experimental allergic encephalomyelitis. Annu. Rev. Immunol. 8:579-621.

22. Seamons, A., Perchellet, A., and Goverman, J. 2003. Immune tolerance to myelin proteins. Immunol. Res. 28:201-221.

23. Fairchild, P.J., Wildgoose, R., Atherton, E., Webb, S., and Wraith, D.C. 1993. An autoantigenic T cell epitope forms unstable complexes with class II MHC a novel route for escape from tolerance induction.
Int. Immunol. 5:1151-1158.

24. Weinhold, B., and Ruther, U. 1997. Interleukin-6 dependent and -independent regulation of the human C-reactive protein gene. Biochem. J. 327:425-429.

25. Breuhahn, K., et al. 2000. Epidermal overexpression of granulocyte-macrophage colony-stimulating factor induces both keratinocyte proliferation and apoptosis. Cell Growth Differ. 11:111-121.

26. Liu, F., Song, Y., and Liu, D. 1999. Hydrodynamicsbased transfection in animals by systemic administration of plasmid DNA. Gene Ther. 6:1258-1266.

27. Nguyen, T.H., and Ferry, N. 2004. Liver gene therapy: advances and hurdles. Gene Ther. 11(Suppl. 1): S76-S84.

28. Liu, G.Y., et al. 1995. Low avidity recognition of self-antigen by $\mathrm{T}$ cells permits escape from central tolerance. Immunity. 3:407-415.

29. Schonrich, G., et al. 1991. Down-regulation of $\mathrm{T}$ cell receptors on self-reactive $\mathrm{T}$ cells as a novel mechanism for extrathymic tolerance induction. Cell. 65:293-304.

30. Anderson, M.S., et al. 2002. Projection of an immunological self shadow within the thymus by the aire protein. Science. 298:1395-1401.

31. Schramm, C., et al. 2003. Impairment of TGF-beta signaling in $\mathrm{T}$ cells increases susceptibility to experimental autoimmune hepatitis in mice. Am.J. Physiol. Gastrointest. Liver Physiol. 284:G525-G535.

32. Lohse, A.W., et al. 1996. Antigen-presenting function and B7 expression of murine sinusoidal endothelial cells and Kupffer cells. Gastroenterology. 110:1175-1181.

33. Hayashi, N., et al. 1999. Kupffer cells from Schistosoma mansoni-infected mice participate in the prompt type 2 differentiation of hepatic T cells in response to worm antigens. J. Immunol. 163:6702-6711.

34. Viñas, O., et al. 2003. Human hepatic stellate cells show features of antigen-presenting cells and stimulate lymphocyte proliferation. Hepatology. 38:919-929.
35. Winau, F., et al. 2007. Ito cells are liver-resident antigen-presenting cells for activating $\mathrm{T}$ cell responses. Immunity. 26:117-129.

36. Herkel, J., et al. 2003. MHC class II-expressing hepatocytes function as antigen-presenting cells and activate specific CD4 T lymphocytes. Hepatology. 37:1079-1085.

37. Wiegard, C., et al. 2007. Defective Thelper response of hepatocyte-stimulated CD4 T cells impairs antiviral CD8 response and viral clearance. Gastroenterology. 133:2010-2018.

38. Wiegard, C., et al. 2005. Murine liver antigen presenting cells control the suppressive activity of $\mathrm{CD} 4^{+} \mathrm{CD} 25^{+}$regulatory $\mathrm{T}$ cells. Hepatology. 42:193-199.

39. Laurence, A., and O'Shea, J.J. 2007. TH-17 differentiation: of mice and men. Nat. Immunol. 8:903-905.

40. Lan, R.Y., et al. 2006. Liver-targeted and peripheral blood alterations of regulatory $\mathrm{T}$ cells in primary biliary cirrhosis. Hepatology. 43:729-737.

41. Longhi, M.S., et al. 2006. Functional study of $\mathrm{CD} 4+\mathrm{CD} 25+$ regulatory $\mathrm{T}$ cells in health and autoimmune hepatitis. J. Immunol. 176:4484-4491.

42. Liu, Y., Teige, I., Birnir, B., and Issazadeh-Navikas, S. 2006. Neuron-mediated generation of regulatory $\mathrm{T}$ cells from encephalitogenic $\mathrm{T}$ cells suppresses EAE. Nat. Med. 12:518-525.

43. Tarbell, K.V., Yamazaki, S., Olson, K., Toy, P., and Steinman, R.M. 2004. CD25+ CD4+ T cells, expanded with dendritic cells presenting a single autoantigenic peptide, suppress autoimmune diabetes. J. Exp. Med. 199:1467-1477.

44. Hogan, B.L.M., Costatini, F., and Lacy, E. 1986. Manipulating the mouse embryo: a laboratory manual. Cold Spring Harbor Laboratory Press. Cold Spring Harbor, New York, USA. 332 pp.

45. Huber, S., et al. 2004. Cutting edge: TGF-beta signaling is required for the in vivo expansion and immunosuppressive capacity of regulatory CD4+CD25+ T cells. J. Immunol. 173:6526-6531. 